\title{
The level of observed physical movement accompanying periodic limb movements measured in a clinical sleep population
}

This article was published in the following Dove Press journal:

Nature and Science of Sleep

\author{
Robert G Hooper \\ The Sleep Center, Scottsdale, AZ, USA
}

Correspondence: Robert G Hooper

The Sleep Center, Post Office Box 4100, Scottsdale, AZ 8526I-4I00, USA

Tel +I 4806570304

Fax + I 4806579129

Email Rhoop@thesleepcenteraz.com
Study objectives: Periodic limb movements (PLMs) are routinely measured during polysomnogram (PSG) testing. During the early years of sleep testing, physical movements were identified and over time, consensus ultimately led to the current definitions of movement disorders including criteria used to measure PLMs on PSG testing. There has been considerable debate about the clinical importance of the PLMs measured during PSG testing. Over the last decade, the author has observed significant variations in the actual visible physical movements observed with a PLM event. This report is the result of work to quantify the amount of movement and the frequency of movements observed among individuals who have PLMs.

Methods/principal findings: Consecutive PSGs performed in a suburban sleep center for an initial diagnosis of a sleep disorder were retrospectively reviewed to identify those with measured PLMs. Of 646 studies on patients $>18$ years, 460 met criteria for inclusion. Visual assessment of movements was carried out on all of those with PLM events measured using American Academy of Sleep Medicine guidelines. The movements were quantified based on the number of extremities observed to move. PLMs were observed in 237 of the 460 studies that met inclusion criteria (52\%). As expected, the PLMs occurred more frequently in older individuals. PLMs occurred with equal frequency in both sexes. Apnea occurred with equal frequency in those with and without observed physical movements. Of those with PLMs, 62\% (147) demonstrated observable physical movements. Significant movements involving three or four extremities occurred in $16 \%$ of individuals with PLMs. No physical movements were observed in $38 \%$.

Conclusion: In this uncontrolled, nonrandom, observational series, visual physical movements with a PLM event identify a unique subset of individuals with PLMs. The presence of any visual movements or more pronounced visual movements involving multiple extremities may represent markers for PLM disorder, for clinically significant PLMs with other disorders, or for other clinical conditions or physiologic variables.

Keywords: periodic limb movements, periodic limb movement disorder, polysomnogram, sleep movements, periodic limb movement index, leg movements, PLM

\section{Plain language summary}

Excessive and abnormal movements occur during sleep. These movements have been identified and classified by physicians. Periodic limb movements (PLMs) are one of these abnormal movements. Although originally identified as a physical movement, now it is measured as a change in electrical muscle activity on sleep testing. PLMs have been recognized for many years and are routinely measured on sleep tests performed in sleep centers. However, their importance in causing sleep symptoms or in disorders of sleep is uncertain. This paper reports the relationship between the measured abnormality on a sleep test and the actually observed physical 
movements. When PLMs are measured on a sleep test, observable movements are not seen in $38 \%$ of those tested, whereas $62 \%$ are observed to move. Major movements involving three or more limbs are made by $16 \%$. Observable movement is a feature that further defines the measurement of this condition. Visual movements may be a marker that identifies patients who have a more serious form of the condition. They could be a marker that identifies those who potentially would benefit from treatment, those who suffer from specific medical conditions causing the PLMs or those who have a unique form of the condition.

\section{Introduction}

The word "movement" appears in the name of the medical condition, periodic limb movement disorder (PLMD), and in the name of the events that are associated with that condition, periodic limb movements (PLMs). According to dictionaries, movement is the "act or process of moving, a change of place or position". However, actual physical movements are seldom reported or measured when PLMs and PLMD are discussed.

As sleep testing became more common beginning in the 1960s and 1970s, a variety of sleep-related movements were recognized, discussed, and studied. ${ }^{1}$ The definition of PLMs evolved from study group reports to consensus papers early in this century. ${ }^{1,2}$ Now the American Academy of Sleep Medicine (AASM) publishes a manual for scoring polysomnogram (PSG) events and a manual of a classification of the disorders of sleep. ${ }^{3,4}$ Currently, the AASM definition of PLMs does not include a requirement for or a description of physical movement, but rather limits the definition to changes seen on electromyogram (EMG) recordings during sleep. The definition of PLMD by the AASM does include descriptions of movements that are seen in the condition and so do early clinical descriptions, reviews, and texts. ${ }^{4-6}$ However, it is not clear how information about or descriptions of the physical movements are to be obtained or documented in the diagnosis and care of patients. Are the movements observed by others, noted by the patient or observed during sleep testing?

If PLMs and PLMD involve abnormal movements during sleep, it seems reasonable that those physical movements should be considered when describing the condition and the presentation of that movement disorder. How often do they occur and of what magnitude are the movements? What role do physical movements have in the condition? Do they relate to symptoms? Do they indicate a level of severity? Do they relate to potential reasons for the PLMs? In order to assess these relationships, quantification and measurement of any physical movement is required.

For 30 years, video technology has been available for the evaluation of sleep in conjunction with PSGs. It has been a part of digital computerized recording systems for over a decade. However, few clinical testing facilities routinely perform comprehensive video recordings during PSGs, and few physicians mention the presence of any movement or the amount of movement associated with PLMs when they interpret the results of PSG tests (personal observations). Yet, it is on PSG testing where PLMs are usually identified.

For much of the past decade, our center has evaluated the amount of physical movement seen visually when PLMs were recorded. A simple system is employed to review the level of movements observed during routine PSG testing for disorders of sleep. The experience has led to this observational report to demonstrate the importance of considering the movements that occur when PLMs are recorded on a PSG. As many clinicians recognize, movements are common, vary greatly, and can be dramatic. ${ }^{4-6}$ This experience argues for the inclusion of movement measurements in the routine evaluation and scoring of PSG tests.

\section{Methods}

Consecutive PSGs performed for a sleep evaluation were reviewed for this report. Full night diagnostic and split night studies were included. Individuals with multiple PSGs were included only once. When patients had two studies, the diagnostic study was selected for inclusion. PSGs from individuals younger than 18 years were excluded. Studies without adequate video recordings were excluded. A video recording of the entire PSG at ten frames per second was required for inclusion. All patients studied had been evaluated by a board certified sleep specialist and were studied for a variety of indications that fell under the AASM guidelines at the time of the studies. ${ }^{7}$ The studies were performed as clinical tests for use in patient care and scored according to AASM standards at the time of study. ${ }^{8}$ This review of data was carried out retrospectively for a 1 -year period when home sleep apnea tests were not performed. The clinical information available for this study included age and sex. Written informed consent to utilize test findings for inclusion in studies of PSG results was obtained from each participant at the time of testing. The Ethical Standards Committee of The Sleep Center approved this study.

The parameter measurements reviewed for this report were made by the clinical staff for the clinical use of physicians and patients. Bilateral anterior tibialis EMG recordings were performed on all studies. PLMs were identified from both left and right anterior tibialis EMGs. A PLM was scored when AASM criteria for a PLM event were met in either or both EMG leads.

PLM frequency was classified by the PLM events per hour of sleep (PLMI). Four levels were assigned and included: 
no PLMs (PLMI <1), minimal (PLMI 1-4.9), mild (PLMI 5-14.9), moderate (PLMI 15-29.9), and severe (PLMI $\geq 30$ ).

The PSGs were evaluated for the portion of the study where the PLMs were observed. The recording was visually divided into thirds. The presence or absence of any series of PLMs within each one-third of the recording was noted. PLMs while awake were not considered when evaluating the portion of the study with PLMs.

The studies were evaluated for the amount or level of movement observed. PLMs, scored by the performing technician and recorded on the PSG, were reviewed by the author who rated the level of movements observed. Movement levels were assigned according to the predominate level of movements observed, not the maximum level of movement. Sections of PLMs identified by EMG were arbitrarily selected for review by the author. The video recorded during the study was played back at a speed of eight times actual recording. The review for visual movements did not include all of the recorded PLMs. The visual movement scoring approach is outlined in Table 1 . The visually observed movements were assigned a movement level of 0 -IV. The definitions of movement levels observed are presented in Table 2. Comparison of patients with apnea to those with no apnea was evaluated with unadjusted ORs with 95\% CIs comparing each level of movement.

\section{Results}

A review of 646 studies identified 460 studies that met criteria for inclusion in this report and were reviewed for PLM events measured by AASM's EMG criteria. They included studies from 167 (36.3\%) females and 293 (63.7\%) males. PLMs were noted in 237 (52\%) of the studies. PLMI >15 were observed in $18.9 \%(87 / 460)$ of our individuals and PLMI $>4.9$ in $31.5 \%$ (145/460). The age distribution shows the population with PSGs reviewed here to be older, with $62 \%$ of these patients being 50 years or older. In these PSGs, both the

Table I Techniques for scoring visual movement

\section{Scoring visual movements}

- Movements were viewed on video recordings of polysomnograms.

- No special instructions were given to technicians or patients regarding sheets, covers or bedclothes.

- The reviewing physician chose selected segments of PLMs to review.

- Duration of review was determined by number of PLMs recorded.

- Video viewing was primarily carried out at a speed of eight times normal with additional review at lower speeds performed as needed for questions about movements.

- Movement classification level was chosen based on predominate movements seen.

Abbreviation: PLMs, periodic limb movements. occurrence of PLMs and the intensity of the PLMI increased with age. The age distribution for the reviewed studies is presented in Table 3. The frequency of PLMs observed was similar between the sexes. Females had a tendency to have PLMs to a lesser extent (lower PLMIs) than males (Table 4).

The profile of the PLMI and the location of PLMs within the study showed the expected. Lower PLMIs $(<15)$ were more frequently seen in only one $(52 \%)$ or two $(28 \%)$ segments of the studies. Higher PLMIs $(\geq 30)$ were seen more commonly throughout all portions of the studies (59\%).

Physical movements were observed in $62 \%$ of the studies that contained PLMs $(147 / 237)$. The level of observed movements are presented in Table 5. The percentage of patients

Table 2 Definitions of level of visual movement observed with measured periodic limb movements

\begin{tabular}{ll}
\hline Definition of Movement Levels \\
\hline Level 0 - & No movements observed \\
Level I - & Movement limited to one extremity \\
& Foot or hand \\
Lower extremity \\
Leg-Knee and below \\
Upper extremity \\
Arm-Forearm and hand \\
Neck or head \\
Movement limited to any combination of two extremities \\
Feet and hands-any two extremities \\
Lower extremity \\
Knee and below-two legs or one leg and one arm \\
Hip with upper and lower leg-unilateral \\
Upper extremity \\
Forearm and hand-two arms or one arm and one \\
lower leg \\
Arm including movement at shoulder-unilateral \\
Neck or head and one extremity \\
Movement limited to any combination of three extremities \\
Feet or hands-any three extremities \\
Upper and lower extremities \\
Knee and below with forearm and hand-any three \\
Upper and lower leg-bilateral \\
Neck or head and two extremities \\
Movement in excess of above
\end{tabular}

Table 3 The age distribution of the 460 patients and the frequency of the identified PLMs

\begin{tabular}{|c|c|c|c|c|}
\hline \multirow[t]{2}{*}{ PLMs identified } & \multicolumn{4}{|l|}{ Age } \\
\hline & $<30$ & $30-49$ & $50-69$ & $>69$ \\
\hline No PLMs & 24 & 71 & 98 & 30 \\
\hline PLMI > I & II & 70 & 99 & 57 \\
\hline Total reviewed & 35 & $14 \mid$ & 197 & 87 \\
\hline No movement, \% & 68.6 & 50.4 & 49.7 & 34.5 \\
\hline Movement observed, \% & 31.4 & 49.6 & 50.3 & 65.5 \\
\hline
\end{tabular}

Abbreviations: PLMIs, periodic limb movements events per hour of sleep; PLMs, periodic limb movements. 
with PLMs at each movement level are presented in Figure 1. Level III or Level IV movements were seen in $16 \%(38 / 237)$ of the patients with measurable PLMs. Level II movements occurred in $18.6 \%$ (44/237), whereas Level I or no movement was seen in the rest of the patients (65.4\% [155/237]). Significant movements were observed at all levels of PLMIs. The movement levels tended to increase with higher PLMIs and with increased age (Table 5). The movements seen can be quite dramatic and involve a significant amount of activity for long durations of the night.

Current proposed standards for the diagnosis of PLMD in the USA require a PLMI of $>15$. In this report, significant levels of movement were seen with PLMI of $<15$ (Table 5). PSG studies with PLMIs $<15$ included 17 with visual movements rated Level III or Level IV. In those PSGs, the significant level of movements could not be explained by apnea as it was only present in $47 \%(8 / 17)$ and the apnea hypopnea index was $<15$ in five of the eight patients with apnea.

Apnea was present to some degree in $63.3 \%(150 / 237)$ of the patients with recorded PLMs and in 64.2\% (143/223) of those who had no PLMs identified. The PLMI decreased as the apnea hypopnea index increased (Table 6). A similar distribution of observed physical movements was seen when apnea was present and when it was absent (Tables 7 and 8).

Table 4 Sex distribution of patients with PLMls as percent of each index level

\begin{tabular}{llllll}
\hline PLMIs & \multicolumn{5}{l}{ Sex of patients with PLMs } \\
\cline { 2 - 6 } & Group & Male & Male \% & Female & Female \% \\
\hline PLMI I-4.9 & 92 & 47 & 32.9 & 45 & 47.9 \\
PLMI 5-14.9 & 58 & 40 & 28.0 & 18 & 19.2 \\
PLMI 15-29.9 & 41 & 24 & 16.7 & 17 & 18.0 \\
PLMI $\geq 30$ & 46 & 32 & 22.4 & 14 & 14.9 \\
Total & 237 & 143 & 100.0 & 94 & 100.0 \\
\hline
\end{tabular}

Abbreviations: PLMls, periodic limb movements events per hour of sleep; PLMs, periodic limb movements.
The observed movements were the same with or without apnea. ORs show that there were no differences in the prevalence of apnea across the five levels of movements between those with apnea and those who had no apnea.

There are other unquantified observations of these visual movements that are important to note. The level of visual movement can vary considerably within one individual's study and even over 5-10 minutes of recorded PLMs within one study (Figure 2). Last, even large movements involving all extremities are not consistently associated with electroencephalogram arousals.

\section{Discussion}

The clinical significance of PLMs has been discussed and debated since EMGs of the extremities became standard practice on PSG testing. Strong arguments and opinions exist supporting the importance and the lack of importance of PLMs. ${ }^{4,9,10}$ Facts are present supporting both sides of the question. Could it be that the definition of PLMs we use for measurement is not complete enough to be able to identify those PLMs, if any, that may be more likely to result in symptoms, clinical problems, or long-term sequelae? The presence of visible physical movements further categorizes PLMs.

PLMs are measured on PSG testing as an EMG abnormality. ${ }^{3}$ Historically and clinically, physical movements are associated with these EMG abnormalities. . $^{1,2,-6}$ While physical movements are described by clinicians and those defining the clinical sleep disorder, the frequency of occurrence and the degree of movement observed has not been reported in a clinical series that the author is able to identify. Discussion of the physical appearance of PLM events seemed to decline and disappear from medical reports with the adoption of EMG criteria to define PLMs. This observational report provides insight into those physical movements. It quantifies the occurrence of visual movements

Table 5 The PLMI measured and the observed movements for the entire population

\begin{tabular}{|c|c|c|c|c|c|c|}
\hline \multirow[t]{2}{*}{ Measured PLMIs } & \multirow[t]{2}{*}{ Group } & \multirow[t]{2}{*}{ None } & \multicolumn{4}{|c|}{ Level of movements observed } \\
\hline & & & Level I & Level II & Level III & Level IV \\
\hline \multicolumn{7}{|l|}{ PLMls $<14.9$} \\
\hline PLMs $<4.9$ & 92 & 51 & 18 & 12 & 8 & 3 \\
\hline PLMs 4.9-14.9 & 58 & 22 & 19 & 11 & 5 & I \\
\hline Subtotal & 150 & 73 & 37 & 23 & 13 & 4 \\
\hline \multicolumn{7}{|l|}{ PLMls $>15$} \\
\hline PLMs 14.9-29.9 & 41 & 9 & 20 & 5 & 5 & 2 \\
\hline PLMs $\geq 30$ & 46 & 8 & 8 & 16 & 7 & 7 \\
\hline Subtotal & 87 & 17 & 28 & 21 & 12 & 9 \\
\hline PLMls $>$ I Total & 237 & 90 & 65 & 44 & 25 & 13 \\
\hline
\end{tabular}

Abbreviations: PLMls, periodic limb movements events per hour of sleep; PLMs, periodic limb movements. 


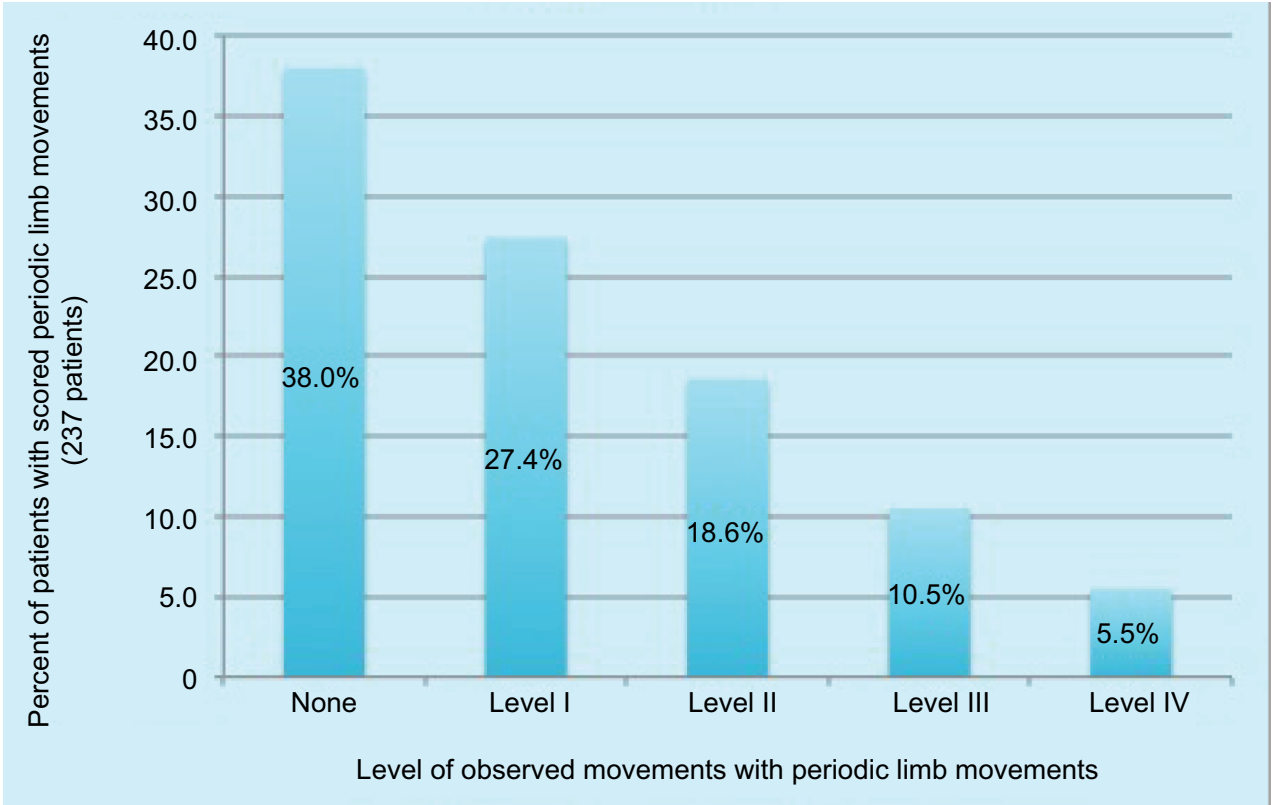

Figure I The observed level of movement in the entire group of patients with periodic limb movements.

Note: The bars represent the percentage of the entire group at each level of observed movements.

Table 6 Relationship between AHI and PLMI

\begin{tabular}{|c|c|c|c|c|c|c|c|c|}
\hline \multirow[t]{3}{*}{ Apnea } & \multicolumn{8}{|c|}{ PLM index } \\
\hline & \multicolumn{2}{|l|}{ PLMI <5 } & \multicolumn{2}{|c|}{ PLMI 5-I4.9 } & \multicolumn{2}{|c|}{ PLMI I5-29.9 } & \multicolumn{2}{|l|}{ PLMI $\geq 30$} \\
\hline & Number & Percent & Number & Percent & Number & Percent & Number & Percent \\
\hline $\mathrm{AHI}<5$ & 32 & 34.8 & 26 & 44.8 & 15 & 36.6 & 14 & 30.4 \\
\hline AHI 5-I4.9 & 26 & 28.3 & 17 & 29.3 & 14 & 34.1 & 18 & 39.1 \\
\hline AHI I5-29.9 & 16 & 17.4 & 8 & 13.8 & 7 & 17.1 & 9 & 19.6 \\
\hline AHI 30-59.9 & 11 & 12.0 & 6 & 10.3 & 4 & 9.8 & 4 & 8.7 \\
\hline $\mathrm{AHI} \geq 60$ & 7 & 7.6 & I & 1.7 & I & 2.4 & 1 & 2.2 \\
\hline Total & 92 & 100 & 58 & 100 & 41 & 100 & 46 & 100 \\
\hline
\end{tabular}

Abbreviations: AHI, apnea hypopnea index; PLMI, periodic limb movement events per hour of sleep; PLMs, periodic limb movements.

Table 7 The level of observed movements in patients without apnea

\begin{tabular}{|c|c|c|c|c|c|c|}
\hline \multirow[t]{2}{*}{ Measured PLMIs } & \multirow[t]{2}{*}{ Group } & \multirow[t]{2}{*}{ None } & \multicolumn{4}{|c|}{ Level of movements observed } \\
\hline & & & Level I & Level II & Level III & Level IV \\
\hline \multicolumn{7}{|l|}{ PLMls $<14.9$} \\
\hline PLMs $<4.9$ & 32 & 14 & 6 & 6 & 5 & 1 \\
\hline PLMs 4.9-I4.9 & 24 & 7 & 10 & 5 & 2 & 0 \\
\hline Subtotal & 56 & 21 & 16 & 11 & 7 & I \\
\hline \multicolumn{7}{|l|}{ PLMls $>15$} \\
\hline PLMs I4.9-29.9 & 15 & 2 & 8 & 2 & 2 & I \\
\hline PLMs $\geq 30$ & 14 & 2 & 3 & 5 & 3 & 1 \\
\hline Subtotal & 29 & 4 & 11 & 7 & 5 & 2 \\
\hline \multirow[t]{2}{*}{ PLMls $>$ I Total } & 85 & 25 & 27 & 18 & 12 & 3 \\
\hline & & $29 \%$ & $32 \%$ & $21 \%$ & $14 \%$ & $4 \%$ \\
\hline
\end{tabular}

Abbreviations: PLMls, periodic limb movements events per hour of sleep; PLMs, periodic limb movements.

in individuals undergoing PSG testing in an uncontrolled suburban clinic test population, by demonstrating the frequency at which visual movements are observed and the magnitude of movements.
PLMs are considered to be an abnormal finding on PSG testing when they reach a numeric threshold defined by a committee of respected sleep physicians. ${ }^{4}$ Healthy controls would not have PLMs by definition. This is a report of PSGs 
Table 8 The level of observed movements in patients with obstructive sleep apnea

\begin{tabular}{|c|c|c|c|c|c|c|}
\hline \multirow[t]{2}{*}{ Measured PLMIs } & \multirow[t]{2}{*}{ Group } & \multirow[t]{2}{*}{ None } & \multicolumn{4}{|c|}{ Level of movements observed } \\
\hline & & & Level I & Level II & Level III & Level IV \\
\hline \multicolumn{7}{|l|}{ PLMls $<14.9$} \\
\hline PLMs $<4.9$ & 60 & 37 & 12 & 6 & 3 & 2 \\
\hline PLMs 4.9-14.9 & 34 & 15 & 9 & 6 & 3 & I \\
\hline Subtotal & 94 & 52 & 21 & 12 & 6 & 3 \\
\hline \multicolumn{7}{|l|}{ PLMls $>15$} \\
\hline PLMs 14.9-29.9 & 26 & 7 & 12 & 3 & 3 & I \\
\hline PLMs $\geq 30$ & 32 & 6 & 5 & 11 & 4 & 6 \\
\hline Subtotal & 58 & 13 & 17 & 14 & 7 & 7 \\
\hline \multirow[t]{2}{*}{ PLMls $>$ I Totals } & 152 & 65 & 38 & 26 & 13 & 10 \\
\hline & & $43 \%$ & $25 \%$ & $17 \%$ & $9 \%$ & $7 \%$ \\
\hline
\end{tabular}

Abbreviations: PLMls, periodic limb movements events per hour of sleep; PLMs, periodic limb movements.

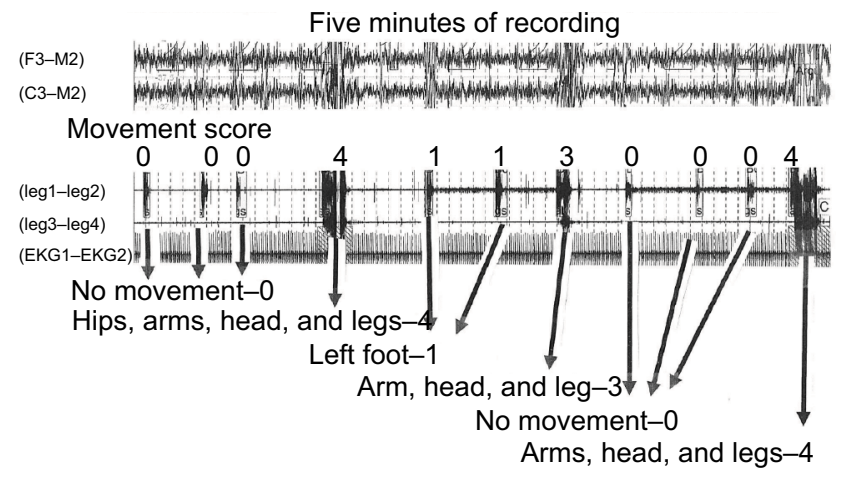

Figure 2 This is an example of the variations in the observed level of movements identified in some individuals.

Notes: It is from a 5-minute segment of a polysomnogram electroencephalogram leads (F3-M2) and (C3-M2), electromyogram leads on left leg(legl-leg2) and right leg (leg3-leg4), and electrocardiogram (EKGI-EKG2).

selected because of the recognized presence of PLMs. It is an attempt to identify the frequency of observed movements and the degree of those movements.

In this group of patients, physical movements were visualized in $62 \%$ of the patients who had PLMI of $>1$ per hour. When movements are observed with a measured PLM event, they can vary from minor to significant. The movements may be limited to the digits of a foot moving a few centimeters or involve one or more extremities moving through a full extension or flexion. Often with minimal movements (Level I-27.4\%) only a slight dorsiflexion of the foot was seen. Significant, sometimes dramatic movements involving multiple extremities (Level III or Level IV) were observed in $16 \%$. The movements visualized were sometimes rapid and quick, whereas other times a similar movement could cover the same distance and involve similar extremities, but be slower and more methodical. Movements occurring in two extremities (Level II-18.6\%) varied. They could be small and slight or more significant.

The presence or absence of visual movements divides the population of PLM patients into two distinct groups that may or may not differ in their clinical characteristics. The level of movements may further define different groups. Those with relatively minor movements (Level I and some with Level II) may have clinical characteristics similar to those with no visual movements, those with greater movements (Level III and Level IV), or have unique features. Certainly, the clinical features of those with greater movements would be anticipated to be different from those with similar PLMI and no movements. Unfortunately, this study's design did not include a review of the clinical features of presentation.

Visual movements increased with the frequency of the PLMs as measured by the PLMI, although elevated PLMIs were seen with no visual movements (Table 5). It should be noted that high levels of visual movements (Level III and Level IV) were observed at all levels of PLMIs. These observations include individuals with significant physical movements with PLMI of under 15 and even $<5$. If the degree of movement is important in a patient's clinical profile or in the long-term clinical effects of PLMD, then individuals with lower PLMIs who have greater movements may be more affected than those with similar or higher PLMIs who have little or no physical movements. PLMD may be a greater clinical issue for patients with higher levels of visual movement than for those with no or limited movements, independent of the level of the PLMI.

The author is unable to identify reports that address the clinical importance of the observed physical movements with PLMs. These observations do not address the question. Being able to see a physical movement does imply a more significant PLM event than a PLM event where a movement is not observed. A PLM event associated with the movement of multiple extremities implies a more significant PLM event than one with less movements. Are they more significant? The addition of a measurement of physical movements to the definition of PLMs would provide a better understanding of the clinical conditions resulting from them. 
The presence of visible movements with PLMs in some patients could imply that not all PLMs, as currently measured on the PSG, are the same. It is generally accepted that PLMs can be the result of a variety of medical conditions, hereditary factors or be idiopathic (PLMD). ${ }^{4}$ Motor activation patterns during PLMs have been shown to be variable. ${ }^{11,12}$ Authors have postulated that PLMs may have different origins in the nervous system. ${ }^{13}$ Of course, movement with a PLM may just be quantitative changes in magnitude of the muscle contractions measured with the EMG. However, our minds should remain open to the possibility that movements may be markers for different etiologies or different neurologic pathways. Further definition and measurement of the physical movements along with correlation of clinical information will be required to address or answer these questions.

Current clinical approaches to the sleep problems produced by PLMs are primarily for symptom control. Evidence has been growing that sleep interruption from PLMs may have long-term negative health effects. ${ }^{14}$ Could the presence of visual movements identify those who would benefit most from treatment? Does the presence of physical movement identify that risk or increase the level of that risk? These are questions that need to be addressed.

All reports have limitations and this one is no exception. It is an uncontrolled, observational, descriptive report of PSG results from a clinical series of symptomatic patients and will by nature not be reliably representative of the general population. However, this series does appear to be similar to results from general population studies regarding the frequency with which PLMs were observed and the age of occurrence. Of the PSGs reviewed for this report, PLMIs $>1$ were found in $31.5 \%$ and PLMIs $>15$ in $18.9 \%$. Estimates of PLMs in the general population vary by country, latitude, and age of the population studied. In one large, general population study in Switzerland, the frequency of measurable PLMs with a PLMI $>15$ was reported in $31 \%$ of men and $26 \%$ of women. Another clinical series reported the frequency of PLMs with PLMI $>15$ in $25 \%$ of their population. ${ }^{15,16}$ PLMs are known to increase with age. ${ }^{1,2,15}$ The frequency of PLMs observed here increased with age.

These are observations of a series of tests performed in a practice setting. The study data were from clinical patient care testing that by necessity involves multiple technicians and scoring staff. Other more defined populations may or may not duplicate the frequency of physical movements reported here. No special efforts were made to be sure to visualize movements. Bedding and patient position might have obscured movements and caused smaller movements to go unnoticed. A consistent method for choosing the portion of the recording where PLMs were present to look for movements, was not employed. In this report only a portion of the recorded PLMs in each PSG were reviewed for visual movements. Reviewing all of the PLMs on a given study might have altered the number of patients observed to move and/or change the number of extremities moved. Of those with PLMs, apnea was present in $63.3 \%$. Apnea may limit the number of measured PLMs. Likewise, the muscle contractions could be isometric or concentric isotonic in nature and, as a result, off-setting muscle contractions or opposing resistance would result in no movements being observed. The evaluation of these possibilities is beyond the scope of these observations. These limitations, for the most part, would have the effect of lessening the level of physical movements observed.

Other limitations are worth noting. The system used here measured only the number of extremities moved, not velocity or strength of those movements. In addition, the visual level of movement reported represents estimated averages of the events viewed as judged by the author, not the maximum or minimum movement observed. What is the best method to measure PLM events and the associated movements? Current standard measurements, including the number of PLM events, the PLMI, and the PLM arousal index, do not incorporate any physical movement into the measurement. A standard system to measure the physical movement should be considered. How should the PLMs reviewed for physical movements be selected? Should all PLMs be scored for physical movement? Should a selected percentage of recorded events be reviewed? Rather than the estimated average level as reported here, should a mathematical average be calculated? Should a profile or histogram of the movements during sleep be created? Should the speed, distance or direction of the movements be measured or quantified in some manner? All of these variables need consideration and study.

\section{Conclusions}

These observations support the use of a method to review for physical movement on PSG testing. The evidence argues for inclusion of the level of physical movements observed with PLMs within the definition of PLMs and PLMD. The findings of significant movements with PLMIs of $<15$ indicated the need for further study of this population. A full night video of each PSG should be standard practice for testing facilities. Interpretation of PSG studies should include a review of the video recording of the patients during PLMs. 
Unfortunately, an observer's assessment will be subjective. However, rather than ignore the physical aspects of the movements, technology should be developed to change these observations into quantitative measurements.

This report demonstrates that visual physical moments are frequent with PLMs but do not occur with all PLMs as measured by current definitions. The movements observed can involve multiple extremities and can be vigorous. These movements occur at all levels of PLMIs but are more common in those of older age and with higher PLMIs. Documenting the presence and the magnitude of visual movements with PLM events serve to further categorize patients with PLMs on PSG testing. The additional stratification and definition of PLMs will aid in our understanding of the clinical conditions with which they are associated.

\section{Disclosure}

The author is the owner and Medical Director of the Sleep Center, PC located in Scottsdale, AZ, USA. The author is solely responsible for this work and has no financial or other conflicts of interest to report.

\section{References}

1. Coleman RC. Periodic movements in sleep (nocturnal myoclonus) and restless legs syndrome. In: Guilleminault C, editor. Sleeping and Waking Disorders: Indications and Techniques. Menlo Park: AddisonWesley; 1982:265-295.

2. Homyak M, Feige B, Riemann D, Voderhoizer U. Periodic leg movements in sleep and periodic limb movements disorder: prevalence, clinical significance and treatment. Sleep Med Rev. 2006;10(3):167-177.
3. aasmnet.org [homepage on the Internet]. The AASM Manual for the Scoring of Sleep and Associated Events: Rules, Terminology and Technical Specifications. American Academy of Sleep Medicine; Available from: http://www.aasmnet.org. Accessed March 13, 2018.

4. American Academy of Sleep Medicine. Sleep related movement disorders. International Classification of Sleep Disorders. 3rd ed. Darien: American Academy of Sleep Medicine; 2014:281-337.

5. Krueger BR. Restless legs syndrome and periodic movements of sleep. Mayo Clin Proc. 1990;65(7):999-1006.

6. Vaughn BV, D’Cruz O. Cardinal manifestations of sleep disorders. In: Kryger MH, Roth T, Dement WC, editors. Principles and Practice of Sleep Medicine. 4th ed. Philadelphia: Elsevier Saunders; 2005:600.

7. Kushida CA, Littner MR, Morgenthaler T, et al. Practice parameters for the indications for polysomnography and related procedures: an update for 2005. Sleep. 2005;28(4):499-521.

8. Iber C,Ancoli-Israel S, Chesson AL Jr, Quan SF. The AASM Manual for the Scoring of Sleep and Associated Events: Rules, Terminology and Technical Specifications. Darien, IL: American Academy of Sleep Medicine; 2007.

9. Hogl B. Periodic limb movements are associated with disturbed sleep. Con. J Clin Sleep Med. 2007;3(1):12-14.

10. Mahowald MW. Periodic limb movements are NOT associated with disturbed sleep. Con. J Clin Sleep Med. 2007;3(1):15-17.

11. Provini F, Vetrugno R, Meletti S, et al. Motor pattern of periodic limb movements during sleep. Neurology. 2001;57(2):300-304.

12. Plazzi G, Vetrugno R, Meletti S, Porvini F. Motor pattern of periodic limb movements in sleep in idiopathic RLS patients. Sleep Med. 2003;3 (Suppl):S31-S34.

13. Clemens S, Rye D, Hochman S. Restless legs syndrome, revisiting the dopamine hypothesis from the spinal cord perspective. Neurology. 2006;67(1):125-130.

14. Walters AS, Rye DB. Review of the relationship of restless legs syndrome and periodic limb movements in sleep to hypertension, heart disease and stroke. Sleep. 2009;32(5):589-597.

15. Haba-Rubio J, Marti-Soler H, Marques-Vidal P, et al. Prevalence and determinants of periodic limb movements in the general population. Ann Neurol. 2016;79(3):464-474.

16. Moro M, Goparaju B, Castillo J, Alameddine Y, Bianchi MT. Periodic limb movements of sleep: empirical and theoretical evidence supporting objective at home monitoring. Nat Sci Sleep. 2016;8:277-289.
Nature and Science of Sleep

\section{Publish your work in this journal}

Nature and Science of Sleep is an international, peer-reviewed, open access journal covering all aspects of sleep science and sleep medicine, including the neurophysiology and functions of sleep, the genetics of sleep, sleep and society, biological rhythms, dreaming, sleep disorders and therapy, and strategies to optimize healthy sleep. The manuscript

\section{Dovepress}

management system is completely online and includes a very quick and fair peer-review system, which is all easy to use. Visit http://www. dovepress.com/testimonials.php to read real quotes from published authors. 